Musées, Patrimoine et Culture scientifiques et techniques

$174 \mid 2017$

novembre-décembre 2017

\title{
Sujet visiteur, Sujet chercheur : questionner les implicites
}

\section{Cora Cohen-Azria}

\section{OpenEdition \\ Journals}

Electronic version

URL: http://journals.openedition.org/ocim/1877

DOI: 10.4000/ocim. 1877

ISSN: 2108-646X

\section{Publisher}

OCIM

\section{Printed version}

Date of publication: 1 November 2017

Number of pages: $32-34$

ISSN: 0994-1908

\section{Electronic reference}

Cora Cohen-Azria, «S Sujet visiteur, Sujet chercheur : questionner les implicites », La Lettre de l'OCIM

[Online], 174 | 2017, Online since 01 November 2018, connection on 25 July 2019. URL : http:// journals.openedition.org/ocim/1877 ; DOI : 10.4000/ocim. 1877

This text was automatically generated on 25 July 2019.

Tous droits réservés 


\title{
Sujet visiteur, Sujet chercheur : questionner les implicites
}

\author{
Cora Cohen-Azria
}

L'article (disponible sur www.ocim.fr, rubrique La Lettre de l'Ocim) montrait notamment comment apprendre à visiter une exposition.

\author{
L'enfant, l'élève, le visiteur \\ ou la formation au musée
}

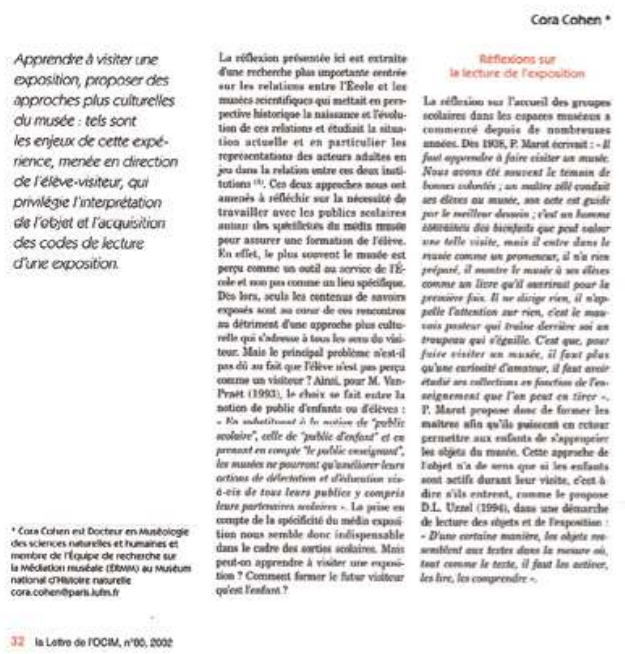

1 En 2002, je m'appuyais sur mon travail de doctorat pour écrire un article sur le statut du visiteur durant les visites scolaires au musée. Il s'agissait alors d'interroger de manière spécifique la formation du sujet (élève et/ou visiteur) dans le cadre des visites scolaires 
dans les expositions scientifiques. Aujourd'hui, l'opportunité m'est offerte de revenir sur cet écrit pour y porter un regard distancié, quelque quinze années plus tard.

2 J'ai ici choisi de travailler sur deux axes qui me semblent fondamentaux tant du point de vue du chercheur que je suis que de l'espace d'écriture qui est le mien aujourd'hui : $L a$ Lettre de l'Ocim. Il s'agit d'interroger dans le sillage de cette première recherche d'une part les relations entre pratiques et recherches et d'autre part les spécificités des disciplines de recherche. Ainsi ce premier axe se structure à partir des échanges que j'ai pu avoir avec les professionnels du musée et de l'école durant différents types de formations (entre autres avec l'Ocim) et de rencontres. Le second permet de revenir sur les disciplines de recherches en jeu et leurs spécificités lorsqu'elles interrogent le visiteur et la visite scolaire.

\section{Élève-visiteur : dialogue entre pratiques et recherches}

3 Il n'existe finalement que peu d'endroits qui permettent de rendre compte des échanges informels vécus durant les stages de formations auxquels on contribue. Pourtant ces moments sont précieux, ils permettent d'avancer, de se poser de nouvelles questions ou de déplacer celles déjà construites. Ce sont quelques points de discussion récurrents ou plus rares qui sont repris ici afin de témoigner de la dynamique de cette rencontre entre pratiques et recherches.

4 À l'époque, je considérais mon travail de recherche de manière très contextualisée. En effet, les grands musées sortaient de leurs rénovations, les expositions étaient le résultat de nouvelles collaborations faisant intervenir d'autres corps de métiers tels que des scénographes par exemple. Il s'agissait alors d'accueillir le public dans des expositions répondant à de nouveaux codes, de nouvelles conceptions de la mise en scène, de la mise en exposition et donc mettant les visiteurs dans une position spécifique. Les objets n'étaient plus toujours exposés pour eux-mêmes, mais étaient mis au service d'un discours scientifique et conceptuel. Ma recherche-action mettant au cœur de son dispositif une formation à devenir visiteur me paraissait alors participer à une démarche transitoire. Avec le temps, il ne serait plus nécessaire d'envisager de telles situations muséales. Toutefois, en m'appuyant sur les sollicitations continues qui me sont faites autour de ce projet et de cette réflexion, je sais aujourd'hui que cette problématique est encore bien d'actualité et qu'elle risque de l'être pour longtemps. La formation du visiteur, le plus souvent implicite ou résultant d'une pratique familiale plutôt que scolaire reste un objet d'interrogation pour les professionnels du musée, mais aussi pour ceux de l'école. Ainsi, durant les formations qui y ont été consacrées jusqu'à présent, j'ai été le témoin ou l'acteur d'échanges permettant de travailler sur les implicites et les évidences partagées au sein des différents corps de métiers. C'est sous forme de questionnement que j'en rends compte ici pour proposer une version ouverte des débats actuels.

5 La place du médiateur muséal reste une question vive. Celui-ci doit-il ou peut-il permettre la rencontre entre des visiteurs et des objets mis en scène ou des visiteurs et des contenus spécifiques ? Selon la réponse qui est donnée, sa place et celle de ceux qu'il accompagne varient. Dans le premier cas, il crée les conditions d'un travail interprétatif des visiteurs qui peuvent construire leurs discours à partir de leurs lectures des présentations. Dans le second, il sert d'intermédiaire entre des contenus choisis, construits en amont de la visite et le groupe. De ce fait, les contenus deviennent des objets d'interrogation incontournables. Lorsque l'on travaille sur les visites d'exposition, ces contenus s'avèrent 
multiples, citons pour exemple les contenus scientifiques préalables à la mise en exposition, les contenus sélectionnés et recomposés dans le but d'écrire l'exposition, les contenus issus des discours des médiateurs, ceux issus des discours des visiteurs... Ils peuvent paraître proches les uns des au-tres, pourtant leur analyse didactique montre une grande variété.

6 Ces réflexions amènent à interroger la parole, sa nature, ainsi que le silence durant les visites. Dans le cadre des visites scolaires dans les expositions, à qui revient-il de parler ? À qui revient-il de faire silence ? À qui revient-il d'écouter ? L'évidence d'une parole associée au guide et d'une écoute associée au groupe-classe est de plus en plus interrogée. Et si c'était le guide qui avait à entendre les propos des visiteurs pour les accompagner, les faire cheminer, pour ajuster la déambulation dans le musée ? Et si c'était le guide qui avait à se taire, à faire silence ? On pourrait m'opposer le fait que cela est pris en compte depuis longtemps et que les guides laissent place aux visiteurs. Toutefois, les études portant sur les groupes scolaires montrent encore le contraire. De plus, le silence n'existe que peu durant les visites scolaires tandis que la valorisation des échanges entre pairs par exemple, hors du réseau groupe classe, demeure encore rare.

7 Cette façon d'interroger les évidences vaut également pour le sens des questions. Est-ce le guide qui questionne le groupe pour faire avancer son propos ou le groupe qui interroge le guide en fonction de ses désirs de savoirs? Souvent les premières impressions amènent à considérer que les questions "voyagent" dans les deux sens : depuis le musée vers les visiteurs et inversement. Pourtant, l'étude des documents d'accompagnement à la visite par exemple nous montre autre chose. Conçus par les acteurs du musée, ils s'appuient le plus souvent sur des questions auxquelles les visiteurs doivent répondre. Pourquoi est-il si rare, de voir sur de tels supports des demandes faites aux visiteurs de construire des questions plutôt que des réponses?

\section{Le statut du visiteur : regards contrastés de chercheurs}

$8 \quad$ Les recherches sur les visites scolaires dans les musées se sont largement développées ces dernières années, et ce, que ce soit en muséologie, en psychologie, en sociologie, en didactique... Les chercheurs de ces différentes disciplines sont parfois amenés à échanger entre eux, toutefois, les objets qu'ils reconstruisent dans leurs recherches s'avèrent, par nature, très contrastés. Pourtant, ces distinctions fondamentales restent souvent implicites surtout lorsque ces recherches sont présentées dans des espaces de vulgarisation. C'est toujours au lecteur de reconstruire l'ancrage du discours, le point de vue de l'auteur, finalement comme le visiteur le fait dans certaines expositions. Lorsque le psychologue regarde le sujet, c'est un enfant qu'il analyse prenant en compte parfois ses émotions, pouvant alors étudier les aspects psycho-cognitifs de la visite. Le sociologue peut envisager les sujets du point de vue de leur appartenance socio-culturelle, et penser la visite comme un moment spécifique de socialisation. Le didacticien prend comme dimension fondamentale les contenus en jeu pour étudier ce avec quoi le sujet doit/peut/ veut faire. Mais, cette dernière approche s'avère, par exemple, différente selon si la recherche est réalisée en France ou au Québec. En effet, en France les disciplines de recherches que sont les didactiques se construisent sur différents espaces disciplinaires (sciences, histoire, arts...), au Québec, à côté de ce découpage, la didactique peut aussi être muséale. 
9 Ainsi, il s'agit de penser les situations au musée comme spécifiques sans que les contenus en jeu n'amènent à envisager une spécificité scientifique supplémentaire. Le sujet didactique n'est pas le même que celui reconstruit par les chercheurs français que sont les didacticiens des différentes disciplines. Cette trop brève présentation des variations montre déjà que la notion de visiteur n'est finalement pas la même selon les disciplines de recherches. Les sujets reconstruits le sont différemment sans que personne ne puisse envisager d'étudier les visiteurs dans leur intégralité, ils sont finalement la somme des sujets reconstruits par les différents regards scientifiques. Et c'est la conscience de cette contextualisation qui permet aux professionnels du musée et de l'école de s'approprier au mieux les contenus des recherches. Cette variation des sujets est donc incontournable, elle est le fait des espaces de recherches et des concepts spécifiques qu'ils permettent de mobiliser.

10 En revanche, les variations institutionnelles peuvent être réinterrogées. En effet, les premières recherches sur l'école et le musée se basaient sur cette dichotomie institutionnelle amenant à penser l'élève et le visiteur de manière contrastée. Les recherches récentes en didactiques propose un appareil conceptuel nouveau envisageant un continuum didactique (Cohen-Azria et Dias-Chiaruttini, 2015) pour analyser la visite scolaire au musée, sortant ainsi des premiers cadres binaires qui aidaient à penser ce qu'étaient l'école et le musée et ce qu'ils n'étaient pas. Cette nouvelle façon d'interroger la visite se centre sur la situation plutôt que sur l'institution évacuant dès lors, l'idée d'une rupture institutionnelle. Cela amène, de fait, d'autres questions méthodologiques, entre autres sur les bornes temporelles de la visite (Cohen-Azria et Dias-Chiaruttini, 2016).

11 Finalement, dans ce propos c'est encore les implicites que j'essaie de débusquer. Il y a quinze ans dans l'article originel, j'interrogeais l'exposition, aujourd'hui les écrits scientifiques, et c'est toujours le sujet (visiteur ou lecteur) qui est au cœur de ces réflexions.

\section{BIBLIOGRAPHY}

Cohen-Azria, C. et Dias-Chiaruttini, A. Analyser les contenus en jeu dans la visite scolaire au Musée : question méthodologiques, in Daunay, B., Fluckiger, C. et Hassan, R. (dir.) Les Contenus d'enseignement et d'ap-prentissage. Approches didactiques, Bordeaux : Presses Universitaires de Bordeaux, 2015, pp. 161-176.

Cohen-Azria, C. et Dias-Chiaruttini, A. La visite scolaire : un espace singulier au croisement de deux institutions, in Cohen-Azria, C., Chopin, M.-P. et Orange, D. (dir.) Questionner l'espace, Questions de temporalité. Les méthodes de recherche en didactiques (4), Villeneuve-d'Ascq ; Presses Universitaires du Septentrion, 2016, pp. 133-148. 


\section{ABSTRACTS}

La rubrique "Quoi de neuf ?" propose un retour sur un article marquant de l'histoire de la revue. À partir d'un corpus d'articles choisis par la rédaction de La Lettre de l'Ocim, les membres du comité des Publications de l'Ocim ont sélectionné plusieurs contributions. Dans cette perspective, il a été demandé à l'auteur ou à un expert du domaine de revisiter la problématique exposée dans l'article à la lueur des changements intervenus, notamment dans les pratiques professionnelles, depuis son écriture et de proposer des éléments prospectifs sur la question.

Cora Cohen-Azria effectue un retour sur l'article "L'enfant, l'élève, le visiteur ou la formation au musée", publié dans le $n^{\circ} 80$ de La Lettre de l'Ocim en mars-avril 2002, qui présentait les enjeux d'une expérience menée vers l'élève-visiteur privilégiant l'interprétation de l'objet et l'acquisition des codes de lectures d'une exposition..

INDEX

Mots-clés: Scolaire, exposition

\section{AUTHOR}

\section{CORA COHEN-AZRIA}

maître de conférences, HdR, en Didactique et muséologie des sciences, Laboratoire ThéodileCIREL EA 4354, université Charles de Gaulle - Lille 3

cora.cohen-azria@univ-lille3.fr 